\title{
Effects of the site distribution and the prior information on the inverted geomagnetic field model: a case study applying the ABIC method to the synthetic datasets
}

\author{
Tadahiro Hatakeyama \\ Information Processing Center, Okayama University of Science, Okayama 700-0005, Japan \\ (Received September 30, 2006; Revised March 24, 2007; Accepted March 29, 2007; Online published July 20, 2007)
}

\begin{abstract}
When we use stochastic inversion and Bayesian modelling in order to obtain geomagnetic field models from paleomagnetic data, there are two major factors controlling the solution: determination of the hyperparameter and the type of the smoothing constraint on the model. To investigate contributions of the factors, we calculated some patterns of inversions from synthetic datasets from ideal and real site distributions. The ABIC (Akaike's Bayesian Information Criteria) minimization method was used to determine the hyperparameter, and then the relationship between the hyperparameter and the ABIC index was demonstrated. Using results of an inversion of synthetic datasets with errors, the most suitable hyperparameters were found for each site distribution, and the good and stable solutions were obtained. However, when number of the sites is few or coverage of the site distribution is not uniform, it is found that the solution is not clearly determined. Moreover, it seems that the solution does not significantly depend on the type of the model constraint.
\end{abstract}

Key words: Geomagnetic field, inversion, ABIC.

\section{Introduction}

In geophysics inverse problems are solved for models. It is quite difficult to solve the geomagnetic, especially paleomagnetic field models. The difficulty is mainly due to restricted acquisition of samples and large errors. Furthermore, paleodirections such as inclination $(I)$ and declination $(D)$ are often obtained in paleomagnetic measurements. Directions without intensity are non-linear functions of the model parameters such as the Gauss coefficients, so that the calculation procedure of the inversion becomes more complicated than the inversion from linear paleomagnetic orthogonal components such as $X, Y$, and $Z$.

Stochastic inversion or Bayesian modelling (e.g., Jackson, 1979; Jackson and Matsu'ura, 1985) is one of the most effective ways for a problem to find a geomagnetic field model from geomagnetic data (Gubbins, 1983, 1984; Gubbins and Bloxham, 1985). Stochastic inversion has been used not only for the historical observed field dataset but also for paleomagnetic data.

One of the main targets solved in this way is the timeaveraged geomagnetic field, TAF (Gubbins and Kelly, 1993; Kelly and Gubbins, 1997; Johnson and Constable, 1995, 1997; Hatakeyama and Kono, 2002). In the TAF problem, global mean magnetic field models are calculated from the long-term averages of paleomagnetic observations at each site.

Another main target of inversion is the continuous magnetic field model which is calculated from paleomagnetic

Copyright (c) The Society of Geomagnetism and Earth, Planetary and Space Sciences (SGEPSS); The Seismological Society of Japan; The Volcanological Society of Japan; The Geodetic Society of Japan; The Japanese Society for Planetary Sciences; TERRAPUB dataset of sediments and archeomagnetic datasets and historical records. Time-depending global field models are smoothly connected with constraint of spline functions. This is much more difficult than solving for TAFs for several reasons: sparse site distribution, less reliable data. Nevertheless, some field models for the past hundreds to thousands years have been obtained by recent development of the reliable historical, sediments, lavas and archeo datasets (Bloxham and Gubbins, 1985; Bloxham and Jackson, 1992; Jackson et al., 2000; Constable et al., 2000; Korte and Constable, 2003, 2005). However we do not yet have enough data to investigate fluctuating fields for long periods as ten thousands or more (Jonson et al., 2003).

The evaluation function to be minimized in the stochastic approaches is different from that in simple least-square method. There is an additional term added to the sum of squares of residual to be minimized. For example, the likelihood function, $S(\boldsymbol{x})$ is,

$$
\begin{aligned}
S(x)=\left(y^{o}-f(x)\right)^{T} & \mathrm{C}_{\mathrm{e}}^{-1}\left(y^{o}-f(x)\right) \\
& +\alpha^{2}\left(x-x^{\#}\right)^{T} \mathrm{C}_{\mathrm{m}}^{-1}\left(x-x^{\#}\right),
\end{aligned}
$$

where $\boldsymbol{x}$ is the vector of the model parameters, e.g. the Gauss coefficients adopted in the global field models, $f$ is the vector function of observation equation, components of $\boldsymbol{y}^{\boldsymbol{o}}$ are the real observations, and $\mathrm{C}_{\mathrm{e}}$ is their covariance matrix.

If we assume that there is no correlation among every observations and every components of the model parameters are independent of each other, two matrices, $C_{e}$ and $C_{m}$, 
become diagonal and Eq. (1) is simplified as following,

$$
S(\boldsymbol{x})=\sum_{i=1}^{N}\left(\frac{y_{i}^{o}-f_{i}(\boldsymbol{x})}{\Delta_{i}}\right)^{2}+\alpha^{2} \sum_{j=1}^{M}\left(\frac{x_{j}-x_{j}^{\#}}{\sigma_{j}}\right)^{2}
$$

where $\Delta_{i}^{2}$ and $\sigma_{j}^{2}$ are respectively diagonal terms of matrices $\mathrm{C}_{\mathrm{e}}$ and $\mathrm{C}_{\mathrm{m}}$. $M$ is number of the model parameters, that means number of rows of $C_{m}$. Actually, we cannot evaluate the function with infinite spherical harmonic degree, $\ell$, so that the calculation should be truncated at $\ell=\ell_{\max }$. The number of the model parameters is $M=\ell_{\max }\left(\ell_{\max }+2\right)$ in scaled linear model or $M=\ell_{\max }\left(\ell_{\max }+2\right)$ in non-scale and nonlinear model.

The second term of Eq. (1) gives the constraint condition on the model, the so-called 'a priori information'. There are two interpretations of $\boldsymbol{x}^{\#}$ and $\mathrm{C}_{\mathrm{m}}$ : (i) probability density: $\boldsymbol{x}^{\#}$ and $\mathrm{C}_{\mathrm{m}}$ represent mean and variance of the probability of model parameters (e.g. Jackson, 1979; Yabuki and Matsu'ura, 1992; Hatakeyama and Kono, 2002), and (ii) physical constraints on the model: e.g. restriction of the field on the core-mantle boundary (CMB) (e.g. Gubbins, 1983; see section 3). Nevertheless, this term shows the penalty on the distance between the solution and the probable model. The first term shows the least-squares residual and $\alpha^{2}(\leq 0)$ is the weight of the second to the first term. The problem reduce to the usual least-squares problem when $\alpha^{2}=0$ and the solution approaches a priori value, $\boldsymbol{x}^{\#}$ when $\alpha \rightarrow \infty$.

In fact, the solution of the inverse problem strongly depends on the magnitude of $\alpha^{2}$. It is very difficult to obtain the most suitable value of $\alpha^{2}$ (Menke, 1989; Parker, 1994). For example, it has been determined with watching the "knee" of the trade-off curve between the data misfit and the model norm (e.g. Gubbins and Bloxham, 1985). The ABIC minimization method (Akaike, 1980) is a powerful tool for determining the best $\alpha^{2}$ in a mathematically objective way. In this study, we test the validity of use of this method in stochastic approaches using synthetic datasets with sparse distribution of sites.

\section{Typical Features of Relationship between $\alpha^{2}$ and $A B I C$}

In the ABIC minimization method (Akaike, 1980) the hyperparameter, $\alpha^{2}$, will be determined maximizing the information entropy in a Bayesian formulation (stochastic process). The hyperparameter is similar to the damping parameter often used in inversion as both show the weight of the model norm term to the data misfit term and they appear to be the same form in the equation. However, its philosophy is different from that of the damping parameter since the hyperparameter is caused by a multiplication of the probability density functions of the data and the model using the Bayes' theorem (Yabuki and Matsu'ura, 1992). In the case of an inverse problem in this study, $\alpha^{2}$ is determined with minimizing a function, $A B I C$, as following,

$$
\begin{aligned}
A B I C\left(\alpha^{2}\right) & \equiv N \ln \left(S\left(\hat{\boldsymbol{x}} ; \alpha^{2}\right)\right)-M \ln \left(\alpha^{2}\right) \\
& +\ln \left\|\mathrm{J}\left(\hat{\boldsymbol{x}} ; \alpha^{2}\right)^{T} \mathrm{C}_{\mathrm{e}}{ }^{-1} \mathrm{~J}\left(\hat{\boldsymbol{x}} ; \alpha^{2}\right)+\alpha^{2} \mathrm{C}_{\mathrm{m}}{ }^{-1}\right\|(3)
\end{aligned}
$$

(detailed derivation of the formula documented in section 2.5 of Yabuki and Matsu'ura, 1992; Appendix of

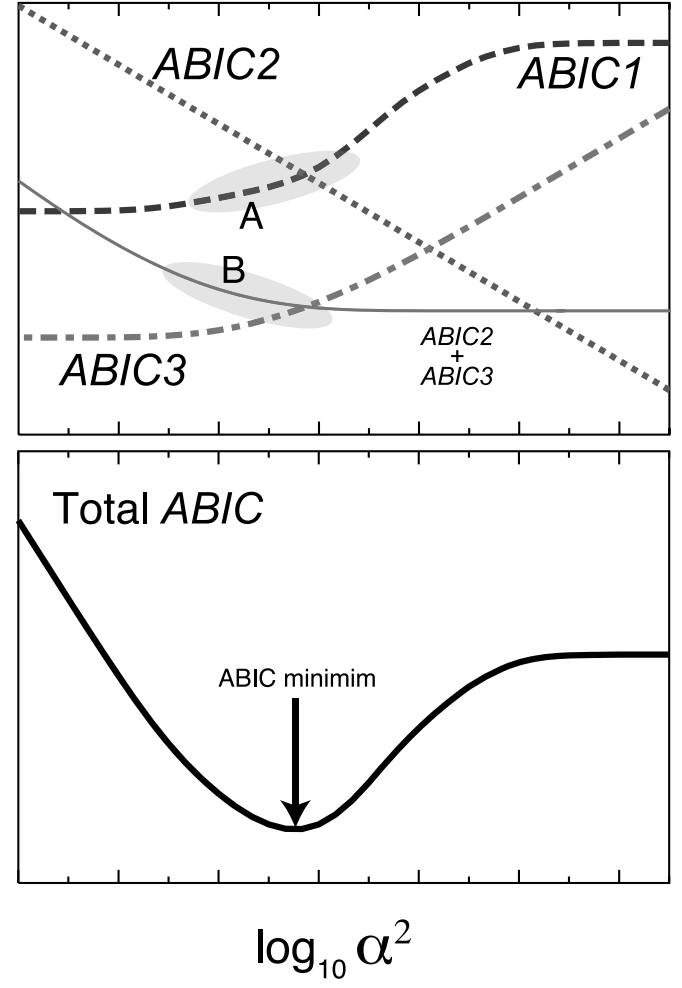

Fig. 1. Typical schematic of $\alpha^{2}-A B I C$ diagram. The upper graph shows the terms of $A B I C$, and the lower graph shows the total $A B I C$. The right and left sides of the diagram correspond to the limits of controlled solution by a priori information and the least-square solution, respectively. The arrow in the diagrams represents the total $A B I C$ minimum point where $\alpha^{2}$ should be regarded as the best one.

Hatakeyama and Kono, 2002; Fukahata et al., 2003). In this equation $\mathrm{J}$ is a Jacobian matrix $\left(\mathrm{J}_{i j}=\partial f_{i} / \partial x_{j}\right)$, and a hat $\left(^{\wedge}\right)$ on $\boldsymbol{x}$ indicates the converged solution with fixed $\alpha^{2}$. The most suitable $\alpha^{2}$ minimizing $A B I C$ will be calculated in numerical process.

The right hand of Eq. (3) has three terms; $A B I C 1=$ $N \ln \left(S\left(\hat{\boldsymbol{x}} ; \alpha^{2}\right)\right), A B I C 2=-M \ln \left(\alpha^{2}\right)$ and $A B I C 3=$ $\ln \left\|\mathrm{J}\left(\hat{\boldsymbol{x}} ; \alpha^{2}\right)^{T} \mathrm{C}_{\mathrm{e}}^{-1} \mathrm{~J}\left(\hat{\boldsymbol{x}} ; \alpha^{2}\right)+\alpha^{2} \mathrm{C}_{\mathrm{m}}{ }^{-1}\right\|$ (Hatakeyama and Kono, 2002). Figure 1 shows typical features of each term and total $A B I C$. ABIC1 is a term derived from the residual. This becomes asymptotically constant at lower and upper limits of $\log \alpha^{2}$, where Eq. (1) approaches the first (the least-square term) and the second (the constraint term), respectively. $A B I C 2$ shows a negative straight line on a semi-logarithmic graph. $A B I C 3$ is also asymptotic to lines at both limits. When $\alpha^{2} \rightarrow \infty$, the curve is close to a positive straight line whose slope is same as the absolute value of $A B I C 2$ 's slope. On the other hand, when $\alpha^{2} \rightarrow 0$ $A B I C 3$ nears a constant value of $\ln \left\|\mathrm{J}^{T} \mathrm{C}_{\mathrm{e}}^{-1} \mathrm{~J}\right\|$. Therefore, $A B I C 2+A B I C 3$ draws a curve which changes from a downside line to a flat line when $\alpha^{2}$ increases.

The value of $\alpha^{2}$ minimizing $A B I C$ depends on a positional relationship between two curves, $A B I C 1$ (shaded region $\mathrm{A}$ in Fig. 1) and $A B I C 2+A B I C 3$ (shaded region B). Parameter determination methods using only the residual curve such as $A B I C 1$ or the least-square misfit (e.g. Parker 1994) are different from the ABIC method. In the ABIC method, the term associated with the first deriva- 


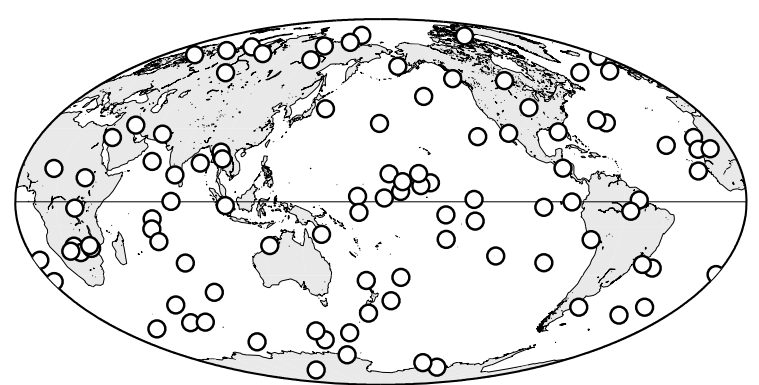

(a) random 100 sites

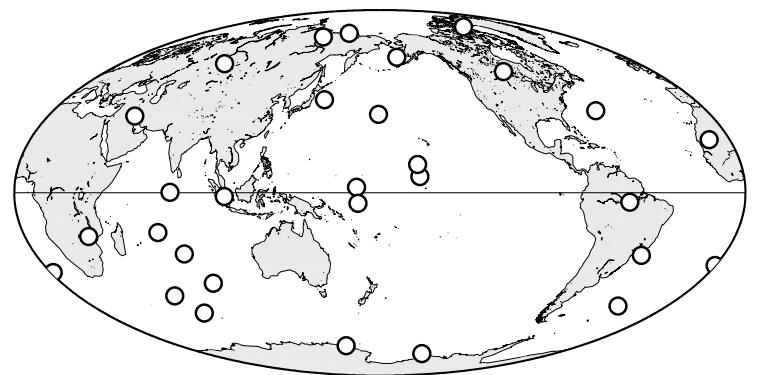

(b) random 30 sites

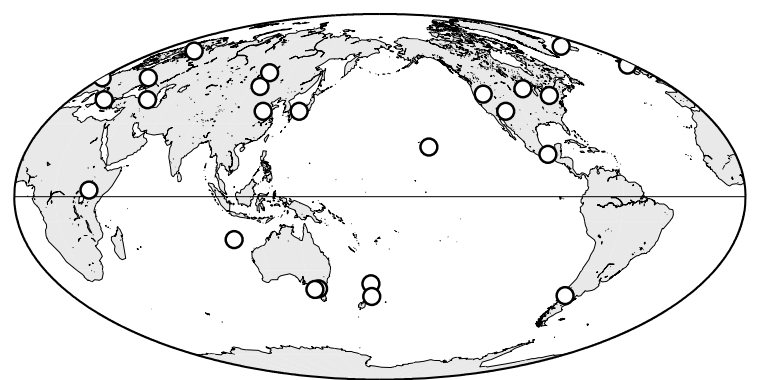

(c) PSVMOD 24 sites

Fig. 2. Maps of site distributions used in this study. (a) random 100 sites (synthetic), (b) random 30 sites (synthetic) and (c) real 24 sites in PSVMOD 1.0 database (Constable et al., 2000).

tion, $A B I C 3$, is used as well as the residual curve, $A B I C 1$. Structure of the $A B I C 3$ term strongly depends on the complexity of the curved surface of $S(\boldsymbol{x})$. This difference may be significant in nonlinear inversions when datasets with large errors are used. For example, if the curved section of $A B I C 2+A B I C 3$ is located in the right side of (higher $\alpha^{2}$ ) the right shoulder of the $A B I C 1$ curve, there is no local minimum in the total $A B I C$. The ABIC minimization method can find such bad case that the quality and quantity of the dataset is insufficient for responsible analysis. If a value of $\alpha^{2}$ locally minimizing $A B I C$ exists it is easy to find numerically.

\section{Settings of Test Calculations}

Inverse calculations of synthetic datasets from three kinds of site distributions and two model constraints are examined in order to investigate the validity of stochastic inversion with the ABIC minimization method and to estimate the effective lower limit of the number of the sites for solving inverse problems of paleomagnetic field models. It seems that the inverse method is successfully performed if the most suitable $\alpha^{2}$ with making $A B I C$ minimum is determined and the solution with the best $\alpha^{2}$ is found, even

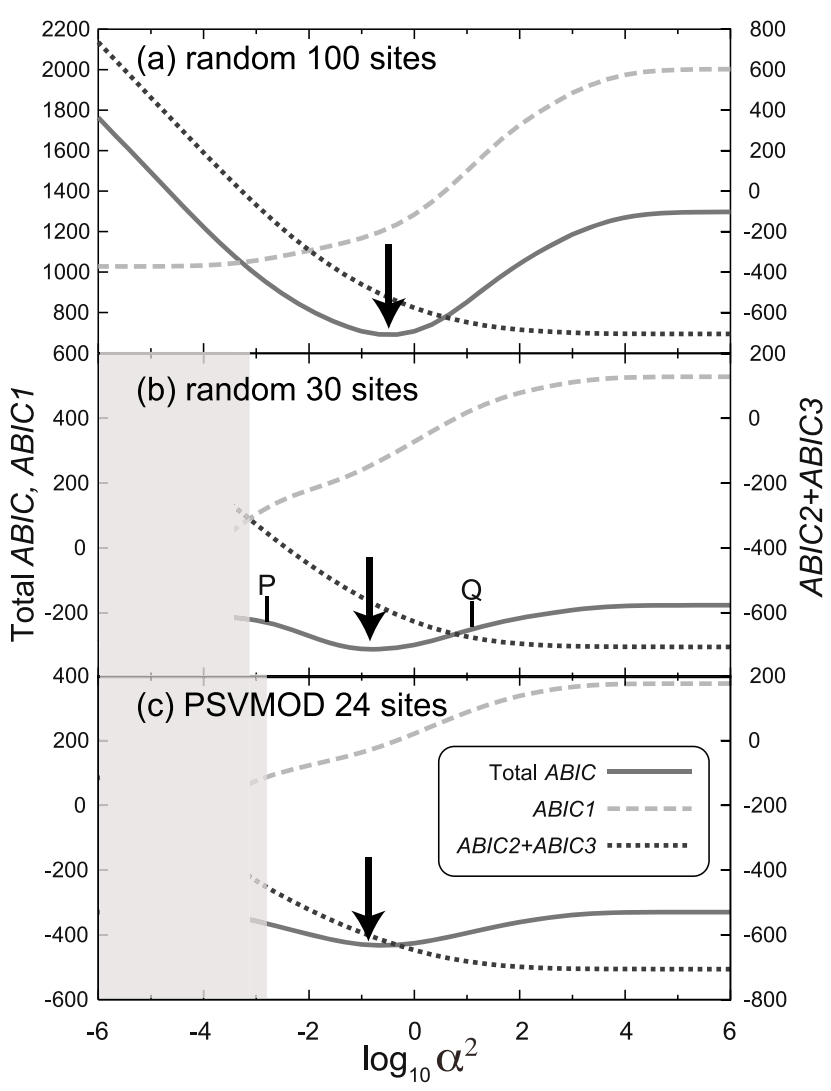

Fig. 3. $\alpha^{2}-A B I C$ diagram to determine the best $\alpha^{2}$ in the ABIC minimization method for the three cases of site distributions, (a) random 100 sites (synthetic), (b) random 30 sites (synthetic) and (c) real 24 sites in PSVMOD database. Values of total $A B I C$ and $A B I C 1$ are shown in the left side axis, and values of $A B I C 2+A B I C 3$ are in the right side axis. Arrows in the diagrams represent the $A B I C$ minima, where the ABIC method certifies that this value of $\alpha^{2}$ is the most suitable. In the shadow regions of (b) and (c), the regularity of the matrices used in the iterative procedures is quite low. Therefore, the solution might be unstable and not unique. a priori information in the calculation used is the type (i).

though the number of sites is quite small.

Reference model and making synthetic dataset

The International Geomagnetic Reference Field of 2005 (IGRF 10th; Maus et al., 2005) was used as the reference model. This can be thought an example of a snapshot of the time-dependent field, which is far from a smooth TAF. We regard this as the "correct" answer and made synthetic datasets for three site distribution patterns and two model constraint conditions below.

First, three orthogonal components $(X, Y$ and $Z)$ at each site were calculated from the reference field model. Next, normal random errors with $1 \sigma$ amplitude of 1000 [nT] were added to each component before calculating directional components, $I$ and $D$, as "direction data with errors". The combination of 1000 [nT] errors in the Cartesian components corresponds to a directional error of $1.5 \sim 3 \mathrm{de}-$ grees.

\section{Common calculation settings}

In all calculations we set the truncation degree of spherical harmonics to 10 . This is likely high enough to solve inverse problems from observations on the Earth's surface (Johnson and Constable, 1995; Hatakeyama and Kono, 2002). All calculations were computed in double precision. 


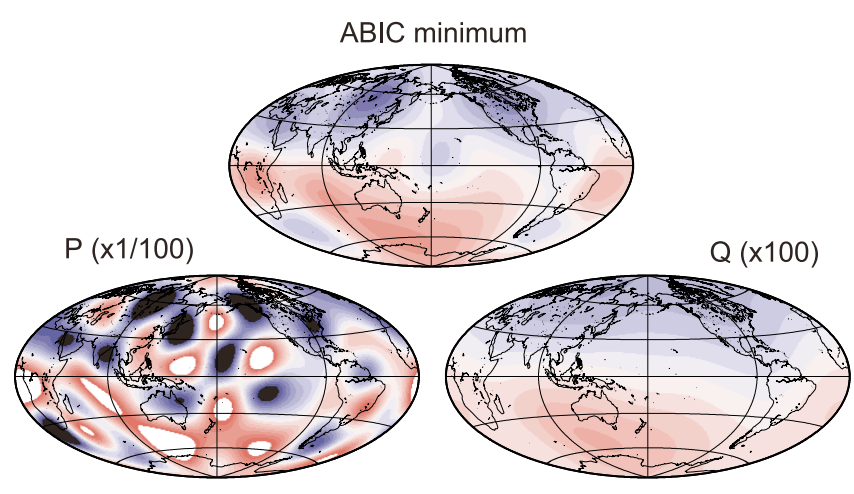

Fig. 4. Solutions in the cases of choice of $\alpha^{2}$, (center) the best value determined by the ABIC minimization method, (left: $\mathbf{P}$ in Fig. 3(b)) 100 times small and (right: $\mathbf{Q}$ in Fig. 3(c)) 100 times large as the best value. It is likely that the best solution of the inversion can be sought with the hyperparameter, $\alpha^{2}$, around the ABIC minimizing. How to see these color maps is documented in Fig. 5.

Iteration is necessary to solve this nonlinear problem and we start from a geocentric axial dipole field, $\boldsymbol{x}_{\text {start }}=g_{1}^{0}$. It is likely that nonlinearity of the calculation is not strong and the correct minima were always found when the iteration started from this starting model.

\section{Site distributions}

Three site distribution patterns were considered (Fig. 2).

(1) random 100 sites

(2) random 30 sites

(3) real 24 sites from the paleomagnetic database PSVMOD 1.0

In cases (1) and (2), sites are randomly distributed on the Earth's surface, which is ideal for spherical harmonic anal-
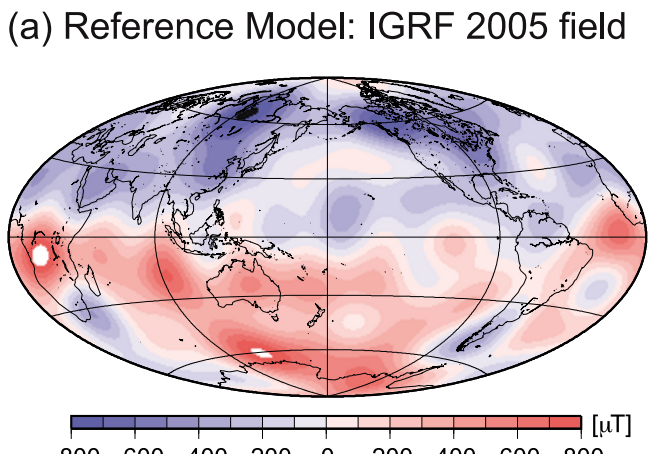

$-800-600-400-200 \quad 0 \quad 200 \quad 400 \quad 600 \quad 800$

(c) random 30 sites

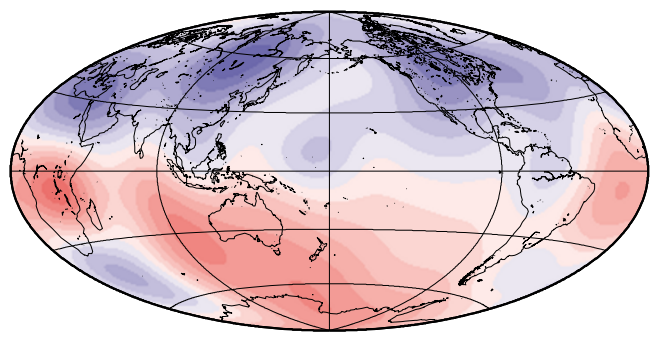

ysis (Mochizuki et al., 1997). The site distribution in case (3) was used by Constable et al. (2000) in their study of the past 3000 years field model. This database includes 17 sites from the north hemisphere, 6 sites from the south hemisphere and a site from the equatorial area. These three site patterns (1), (2) and (3) are examples assuming cases that (1) sites are densely located on surface, (2) sites are sparse but not biased and (3) sites are sparse and biased, which is realistic for paleomagnetic problems.

Constraints on model (a priori information)

Two conditions as a priori information on the model were dealt with.

(i) Paleosecular variation (PSV) model: Each Gauss coefficient has normal distribution with mean $x_{j}^{\#}$ and variance $\sigma_{j}$ (Hatakeyama and Kono, 2002). This PSV model was based on the Giant Gaussian Process (Constable and Parker, 1988), that the field becomes smooth on the CMB. This attitude follows the original philosophy of Bayesian modeling and stochastic inversion (Jackson, 1979; Jackson and Matsu'ura, 1985).

(ii) Physical condition that the ohmic heating on the CMB is to be minimized (Gubbins, 1983; Korte and Constable, 2003; etc.): From this point of view, the second term is not regarded as the probability density. It is not necessary that this term is not in a quadratic form of model parameters. In this case the constrains high degree components slightly stronger than the condition of (i).

Anyway, the constraint must be selected as high degree components of the solution converge, so that it is a representation of conditions at the CMB (Gubbins, 1983). Orders of magnitude of $\sigma_{j}^{2}$ in Eq. (2)

\section{(b) random 100 sites}

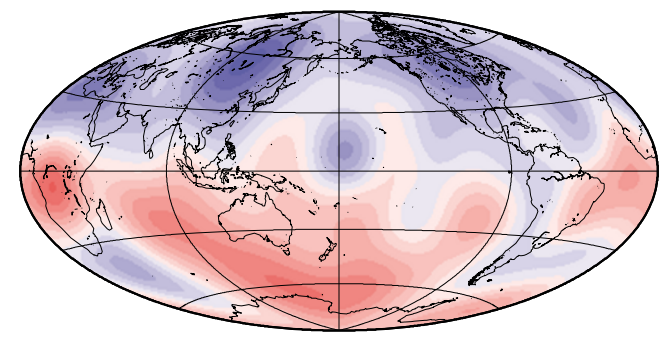

(d) PSVMOD 24 sites

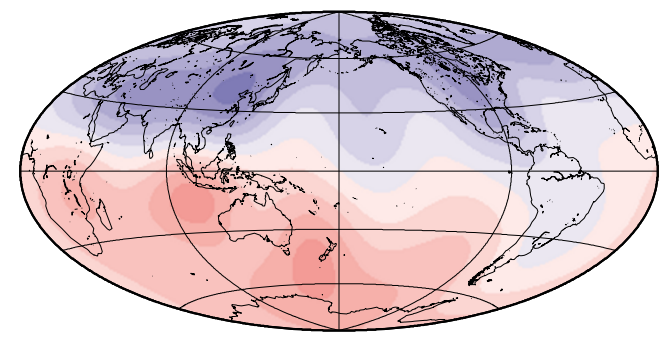

Fig. 5. Solutions with the ABIC minimization method. (a) reference model, the expected one, (b) $\sim(\mathrm{d})$ are from the synthetic datasets for three type of site distributions given in Fig. 2. Color maps show the radial component of the magnetic field, $B r$, on the core surface in order to clearly discriminate a trivial difference between the models. Radius ratio of the core to the Earth is $R_{\text {core }} / R_{\text {earth }}=0.544$. For calculations (b), (c) and (d) the type (i) of prior information is used. 
Table 1. The Gauss coefficients of the reference model and the solutions. "Sol" columns mean the values of the solution. "Std" columns are the square root of the diagonal terms of the model covariance matrices which shows the model accuracy. Here values of the degree $\ell \leq 3$ are demonstrated, but they were $\ell \leq 10$ in all calculations. The geocentric axial dipole component, $g_{1}^{0}$, is fixed in all calculations. Unit of field is [nT].

\begin{tabular}{c|r|rc|rc|rc} 
& IGRF & \multicolumn{2}{|c|}{ Random 100 sites } & \multicolumn{2}{|c|}{ Random 30 sites } & \multicolumn{2}{|c}{ PSVMOD 24 sites } \\
& (Model) & \multicolumn{1}{|c|}{ Sol } & Std & \multicolumn{1}{c}{ Sol } & Std & \multicolumn{1}{c}{ Sol } & Std \\
\hline$g_{1}^{0}$ & -29556.8 & -29556.8 & - & -29556.8 & - & -29556.8 & - \\
$g_{1}^{1}$ & -1671.8 & -1649.0 & $(109.3)$ & -1237.3 & $(218.3)$ & -1263.8 & $(461.4)$ \\
$h_{1}^{1}$ & 5080.0 & 4825.4 & $(114.6)$ & 5224.8 & $(272.6)$ & 5354.1 & $(359.4)$ \\
$g_{2}^{0}$ & -2340.5 & -2245.0 & $(110.0)$ & -2066.9 & $(288.6)$ & -1401.0 & $(484.4)$ \\
$g_{2}^{1}$ & 3047.0 & 2994.6 & $(140.7)$ & 2686.6 & $(250.0)$ & 1755.9 & $(544.0)$ \\
$h_{2}^{1}$ & -2594.9 & -2622.4 & $(150.9)$ & -2376.9 & $(290.1)$ & -3102.5 & $(417.0)$ \\
$g_{2}^{2}$ & 1656.9 & 1602.9 & $(80.6)$ & 1757.9 & $(234.4)$ & 1207.2 & $(244.6)$ \\
$h_{2}^{2}$ & -516.7 & -624.7 & $(80.8)$ & -793.0 & $(184.5)$ & 366.3 & $(322.7)$ \\
$g_{3}^{0}$ & 1335.7 & 973.1 & $(113.7)$ & 1286.2 & $(235.8)$ & 1075.1 & $(319.8)$ \\
$g_{3}^{1}$ & -2305.3 & -2241.4 & $(104.8)$ & -2468.6 & $(187.7)$ & -691.4 & $(449.9)$ \\
$h_{3}^{1}$ & -200.4 & -315.9 & $(111.0$ & -456.2 & $(202.7)$ & -565.4 & $(332.2)$ \\
$g_{3}^{2}$ & 1246.8 & 1214.8 & $(100.4)$ & 1267.7 & $(235.4)$ & 954.5 & $(220.1)$ \\
$h_{3}^{2}$ & 269.3 & 358.1 & $(103.5)$ & 455.9 & $(221.6)$ & -409.4 & $(281.3)$ \\
$g_{3}^{3}$ & 674.4 & 580.9 & $(65.2)$ & 562.1 & $(188.4)$ & 382.9 & $(198.9)$ \\
$h_{3}^{3}$ & -524.5 & -521.2 & $(65.5)$ & -579.8 & $(161.9)$ & 191.2 & $(277.2)$
\end{tabular}

are (i) $\sigma_{j}^{2} \sim \mathcal{O}\left(\ell^{-2}\left(R_{\text {core }} / R_{\text {earth }}\right)^{2 \ell}\right)$ and (ii) $\sigma_{j}^{2} \sim$ $\mathcal{O}\left(\ell^{-3}\left(R_{\text {core }} / R_{\text {earth }}\right)^{2 \ell}\right)$, respectively. It is expected that in case (ii) high degree components of the solution are much suppressed and smoother than those in case (i), as $\left.\left[\sigma_{10} / \sigma_{1}\right]_{(\mathrm{ii})} /\left[\sigma_{10} / \sigma_{1}\right)_{(\mathrm{i})}\right] \sim 2$.

\section{Results and Discussions}

\subsection{Stability of calculation and choice of the best $\alpha^{2}$}

Here we show the results of determination of the "best" values for the hyperparameter, $\alpha^{2}$ using the ABIC method.

Figure 3 shows the typical relationship between $\alpha^{2}$ and $A B I C$ by scanning $\alpha^{2}$ and solving the stochastic inversion at each point. The ABIC minimization method could determine a suitable hyperparameter and a good field model was solved in each case. Actually, when we adopted smaller or larger $\alpha^{2}$ than the "best value", rougher (error overestimated) or smoother (error underestimated) solutions were obtained (Fig. 4).

In case (1) (100 random sites) the solutions were found in the whole range of $\alpha^{2}$, because the dataset has sufficient information and the matrix to be inverted keeps regularity even if $\alpha^{2}$ becomes small. Therefore, we can easily find a local minimum of $A B I C$.

On the other hand, even in the scarce sites cases of (2) and (3), local minima of $A B I C$ could be determined. However when we seek the minimum value of the likelihood function, Eq. (1), with using somewhat smaller $\alpha^{2}$, regularity of the inverse matrix collapses and stable solution cannot be found even in double precision calculations (shaded regions in Fig. 3(b) and (c)).

It is supposed that the most suitable $\alpha^{2}$ determined by the ABIC method would enter in this region when the data are worse or the number of sites is less. In those cases, we should not choose the parameter just above the collapse of the calculation with our subjective view. We have to

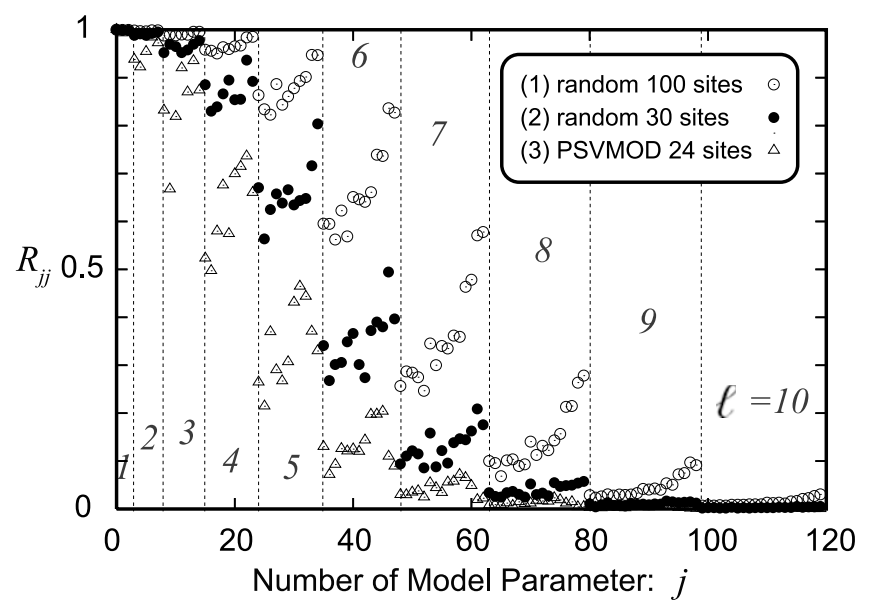

Fig. 6. Diagonal terms of the resolution matrix, $\mathrm{R}$, which shows the model resolution of the components, $x_{j}$. The larger $R_{j j}$ is, the better model resolution and reproductivity is.

conclude 'it is impossible to calculate because the dataset is either bad or insufficient. a model is not found'.

\subsection{Solutions}

Here we compare solutions of calculations with different types of site distribution and prior information.

Figure 5 shows the reference model and solutions of the inverse problems on the core surface. The main characteristics of the field are similar to each other. However, details of the field structures are different, which is caused by differences in the degree components. $\mathrm{Br}$ components of fields (b), (c) and (d) are smoother than that of (a), where high degree components of the solutions are much suppressed by the prior information on the model in the stochastic inversion. The solution becomes smooth as much as site distribution is sparse. The reason is likely that restricted information depending on the number and location of sites 


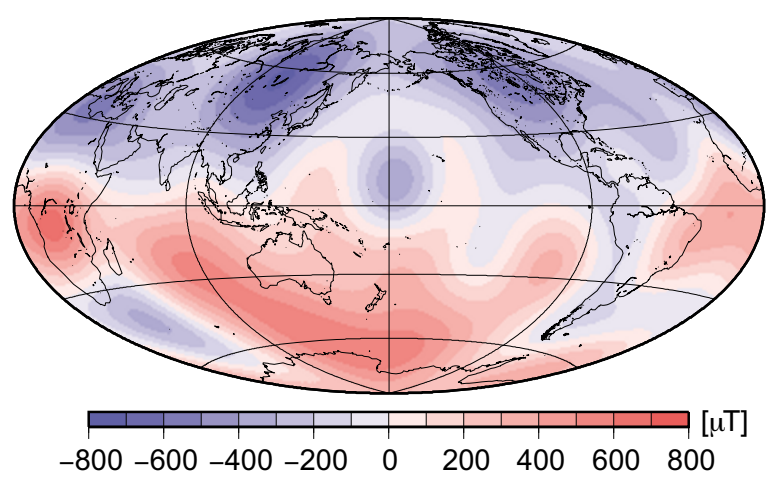

Fig. 7. Solution with using the other type of constraints (ii) on the model to compare with Fig. 5(b).

is redistributed to the components of spherical harmonics. The second term in the right hand of Eq. (1) cuts down the high degree components.

However, higher degree components of the magnetic field are not significantly seen on the Earth's surface, because small structures of the magnetic field on the CMB attenuates according to the order of $\left(R_{\text {core }} / R_{\text {earth }}\right)^{\ell+1}$ (Langel and Estes, 1982). Therefore, paleomagnetic observations are not sensitive to the high degree components of the field and it is supposed that there is no significant difference in characteristics between these field models.

Inverse calculations for (1) random 100 sites and (2) random 30 sites cases could successfully determine components of degree $\ell \leq 6$ and $\ell \leq 4$, respectively (Table 1 ). The values of those components lie in the range defined by the covariance matrix and the reference values. In case (3) of 24 real sites, on the other hand, some components, e.g. $g_{2}^{0}, g_{2}^{1}$ and $g_{3}^{1}$, even in low degree of $\ell=2$ or 3 are not well solved. This may indicate that not only enough data (sites) but also a good distribution of sites is necessary to obtain a good solution. It is suggested that these components in spherical harmonics are almost in the shadow of the observation function, $f(x)$.

The model resolution matrix, $\mathrm{R}=\left(\mathrm{J}^{T} \mathrm{C}_{\mathrm{e}}{ }^{-1} \mathrm{~J}+\right.$ $\left.\alpha^{2} \mathrm{C}_{\mathrm{m}}{ }^{-1}\right)^{-1} \hat{\jmath}^{T} \mathrm{C}_{\mathrm{e}}{ }^{-1} \hat{\mathrm{J}}$, also indicates that there are some uncertain components in the inversion procedure (Fig. 6). The diagonal terms of $\mathrm{R}$ show reproductivity and reliability of the model parameters (Bloxham et al., 1989). It is generally supposed that a higher order, $m$, component has better resolution than lower order components in each degree $\ell$ (Kelly and Gubbins, 1997; Hatakeyama and Kono, 2002). This characteristic can be seen in the results of widely distributed sites case (1) and (2). Nevertheless, in the case of (3) real 24 sites, some low degree components such as $g_{3}^{1}$ have very low resolution. It is indicated that a sparse and irregular distribution (Fig. 1(c)) causes ill-determined elements in the inversion with spherical harmonic expansion.

Figure 7 shows the solution using another prior information (ii) on the model parameters. Its main feature is not significantly different from that of the calculation using condition (i). It is likely that such difference of condition does not influence a solution very much.

The results in this study indicate that the ABIC minimization method in stochastic inversion for paleomagnetic field models determines the hyperparameter, $\alpha^{2}$ for a good solution.

However, when the number of site and data is small, we face difficulty in seeking the best $\alpha^{2}$ to minimize $A B I C$. Moreover, regions where no data exists have negative effects even on the low spherical harmonic degree components in the solution. There is little difference of solutions for the two types of a priori information used in this paper.

At least 30 sites in the world is necessary to obtain a good geomagnetic model derived by the stochastic inversion even if the quality of the data is same as that in this study (the error used in this study is thought to be moderately low for sediment data). Otherwise, even low degree components are smooth and indistinct as specified by the prior information. It is also important that data should come from sites widely covered the world. In reality, we have a serious problem that there are very few data from southern hemisphere. However, it is expected that good samples of deep-sea sediments from southern hemisphere sites will be provided by future drilling programs.

There is another critical problem that declination is unreliable in many paleomagnetic data because of rotation of the core samples and other reasons. If we use a dataset in which only inclination is available many sites are necessary for analysis (Kono, 1976). Unfortunately, the present number of paleomagnetic sites is probably insufficient for analysis over the past ten thousand years.

Acknowledgments. I thank Y. Fukahata, H. Mizutani and M. Kono for useful discussions. I also appreciate D. Gubbins and an anonymous reviewers' comments to improve the manuscript. This research was partially supported by the Ministry of Education, Science, Sports and Culture, Grant-in-Aid for Young Scientists, No. 16740258.

\section{References}

Akaike, H., Likelihood and the Bayes procedure, in Bayesian Statistics, edited by J. M. Bernardo, M. H. DeGroot, D. V. Lindley, and A. F. M. Smith, University Press, Valencia, 143-166, 1980.

Bloxham, J. and D. Gubbins, The secular variation of Earth's magnetic field, Nature, 317, 777-781, 1985.

Bloxham, J. and A. Jackson, Time-dependent mapping of the magnetic field at the core-mantle boundary, J. Geophys. Res., 97, 19537-19563, 1992.

Bloxham, J., D. Gubbins, and A. Jackson, Geomagnetic secular variation, Phil. Trans. R. Soc. Lond. A., 329, 415-502, 1989.

Constable, C. G. and R. L. Parker, Statistics of the geomagnetic secular variation for the past 5 m.y., J. Geophys. Res., 93, 11569-11581, 1988.

Constable, C. G., C. L. Johnson, and S. P. Lund, Global geomagnetic field models for the past 3000 years: transient or permanent flux lobes?, Phil. Trans. R. Soc. Lond. A, 358, 991-1008, 2000.

Fukahata, Y., Y. Yagi, and M. Matsu'ura, Waveform Inversion for seismic source processes using ABIC with two sorts of prior constraints: Comparison between proper and improper formulations, Geophys. R. Lett., 30, 1-4, doi:10.1029/2002GL016293, 2003.

Gubbins, D., Geomagnetic field analysis - I. stochastic inversion, Geophys. J. R. astr. Soc., 73, 641-652, 1983.

Gubbins, D., Geomagnetic field analysis - II. secular variation consistent with the perfectly conducting core, Geophys. J. R. astr. Soc., 77, 753$766,1984$.

Gubbins, D. and J. Bloxham, Geomagnetic field analysis - III. Magnetic fields on the core-mantle boundary, Geophys. J. R. astr. Soc., 80, 695$713,1985$.

Gubbins, D. and P. Kelly, Persistent patterns in the geomagnetic field over the past 2.5 Myr, Nature, 365, 829-832, 1993.

Hatakeyama, T. and M. Kono, Geomagnetic field model for the last 5 My: time-averaged field and secular variation, Phys. Earth Planet. Inter., 
133, 181-215, 2002.

Hongre, L., G. Hulot, and A. Khokhlov, An analysis of the geomagnetic field over the past 2000 years, Phys. Earth Planet. Inter., 106, 311-335, 1998.

Jackson D. D., The use of a priori data to resolve non-uniqueness in linear inversion, Geophys. J. R. astr. Soc., 57, 137-157, 1979.

Jackson, D. D. and M. Matsu'ura, A Bayesian approach to nonlinear inversion, J. Geophys. Res., 90, 581-591, 1985.

Jackson, A., A. R. T. Jonkers, and M. Walker, Four centuries of geomagnetic secular variation from historical records, Phil. Trans. R. Soc. Lond. A, 358, 957-990, 2000.

Johnson, C. L. and C. G. Constable, The time-averaged geomagnetic field as recorded by lava flows over the past $5 \mathrm{Myr}$, Geophys. J. Int., 122, 488-519, 1995.

Johnson, C. L. and C. G. Constable, The time-averaged geomagnetic field: global and regional biases for 0-5 Ma, Geophys. J. Int., 131, 643-666, 1997.

Johnson, C. L., C. G. Constable, and L. Tauxe, Mapping longterm changes in Earth's magnetic field., Science, 300, 2044-2045, doi:20.1126.science.1032007, 2003.

Kelly, P. and D. Gubbins, The geomagnetic field over the past $5 \mathrm{Myr}$, Geophys. J. Int., 128, 315-330, 1997.

Kono, M., Uniqueness problems in the spherical harmonic analysis of the geomagnetic field direction data, J. Geomag. Geoelectr., 28, 11-29, 1976.

Korte, M. and C. G. Constable, Continuous global geomagnetic field models for the past 3000 years, Phys. Earth Planet. Inter., 140, 73-89, doi:10.1016/j.pepi.2003.07.013, 2003.
Korte, M. and C. G. Constable, Continuous geomagnetic field models for the past 7 millennia: 2. CALS7K, Geochem. Geophys. Geosys., 6, doi:10.1029/2004GC000801, 2005.

Langel, R. A. and R. H. Estes, A geomagnetic field spectrum, Geophys. R. Lett., 9, 250-253, 1982.

Maus, S., S. Macmillan, T. Chernova, S. Choi, D. Dater, V. Golovkov, V. Lesur, F. Lowes, H. Luhr, W. Mai, S. McLean, N. Olsen, M. Rother, T. Sabaka, A. Thomson, T. Zvereva, and International Association of Geomagnetism, Aeronomy (IAGA), Division V, Working Group VMOD, The 10th generation international geomagnetic reference field, Phys. Earth Planet. Inter., 151, 320-322, 2005.

Menke, W., Geophysical Data Analysis: Discrete Inverse Theory, Rev. Ed., 289 pp., Academic Press, 1989.

Mochizuki, E., Y. Yokoyama, I. Shimizu, and Y. Hamano, Spherical harmonic analysis in terms of unevenly distributed observation points and its applications to geomagnetic data, J. Geomag. Geoelectr., 49, 10131033, 1997.

Parker, R. L., Geophysical Inverse Theory, 386 pp., Princeton Univ. Press, 1994.

Yabuki, T. and M. Matsu'ura, Geodetic data inversion using a Bayesian information criterion for spatial distribution of fault slip, Geophys. $J$. Int., 109, 363-375, 1992.

T. Hatakeyama (e-mail: hatake@center.ous.ac.jp) 